\title{
Management of corneal bee sting
}

This article was published in the following Dove Press journal:

Clinical Ophthalmology

| December 201 |

Number of times this article has been viewed

\section{Hassan Razmjoo ${ }^{1,2}$}

Mohammad-Ali Abtahi',2,4

Peyman Roomizadeh ${ }^{1,3}$

Zahra Mohammadi ${ }^{1,2}$

Seyed-Hossein Abtahi',3,4

'Medical School, Isfahan University of Medical Sciences (IUMS);

${ }^{2}$ Ophthalmology Ward, Feiz Hospital, IUMS; ${ }^{3}$ Isfahan Medical Students Research Center (IMSRC), IUMS; ${ }^{4}$ Isfahan Ophthalmology Research Center (IORC), Feiz Hospital, IUMS, Isfahan, Iran

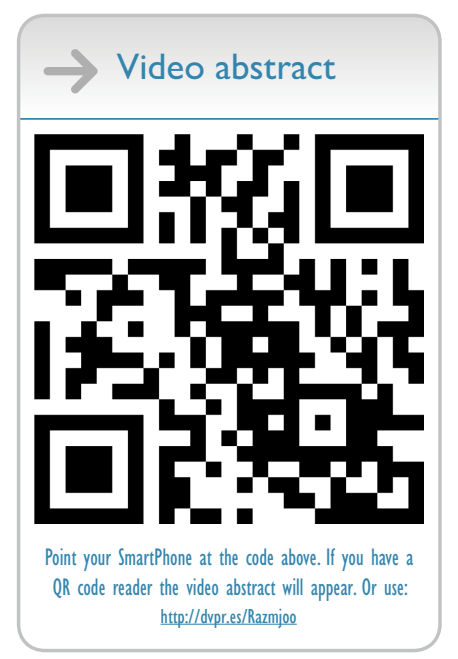

Correspondence: Seyed-Hossein Abtahi S.H.A. Official Research Center of Neurological-Ophthalmological Sciences (SHARNOS Co), No 9, Boroomand,

Seyed-Alikhan, Chaharbagh Abbasi,

Isfahan 8I448-I458I, Iran

Tel +98913 4098036

Fax +98 3II 2643588

Emailshf.abtahi@yahoo.com
Abstract: Corneal bee sting is an uncommon environmental eye injury that can result in various ocular complications with an etiology of penetrating, immunologic, and toxic effects of the stinger and its injected venom. In this study we present our experience in the management of a middleaged male with a right-sided deep corneal bee sting. On arrival, the patient was complaining of severe pain, blurry vision with acuity of 160/200, and tearing, which he had experienced soon after the injury. Firstly, we administered conventional drugs for eye injuries, including topical antibiotic, corticosteroid, and cycloplegic agents. After 2 days, corneal stromal infiltration and edema developed around the site of the sting, and visual acuity decreased to 100/200. These conditions led us to remove the stinger surgically. Within 25 days of follow-up, the corneal infiltration decreased gradually, and visual acuity improved to 180/200. We suggest a two-stage management approach for cases of corneal sting. For the first stage, if the stinger is readily accessible or primary dramatic reactions, including infiltration, especially on the visual axis, exist, manual or surgical removal would be indicated. Otherwise, we recommend conventional treatments for eye injuries. Given this situation, patients should be closely monitored for detection of any worsening. If the condition does not resolve or even deteriorates, for the second stage, surgical removal of the stinger under local or generalized anesthesia is indicated.

Keywords: bee sting, stinger, cornea, removal, management, surgery

\section{Introduction}

Corneal bee sting is an uncommon environmental eye injury that can result in various ocular complications, including corneal epithelial defect, corneal stromal infiltration, endothelial cell loss, glaucoma, cataract, anterior uveitis, optic neuritis, and even permanent vision loss. ${ }^{1-6}$ The etiology of these complications is related to penetrating, immunologic, and toxic effects of the stinger and its injected venom through the cornea. ${ }^{1,7}$

Unfortunately, due to the rarity of this condition, there is no clear guideline for management of affected individuals. Overall, in the current literature, the clinical approach to corneal bee stings remains controversial, ranging from conservative to surgical choices. ${ }^{4,8}$ On one hand, in some studies, cases were treated only with conventional therapies for eye injury and the stinger remained in situ. ${ }^{3,7}$ On the other hand, a surgical approach was chosen to remove the retained stingers from the cornea in a number of cases. ${ }^{6,89}$ However, in such instances, the exact surgical procedure is not well described.

In the meantime, there is an essential need for a uniform therapeutic algorithm that can summarize the results of sparse studies in the field. In this report we present 
our experience in the management of a patient with a corneal bee sting. We aim to describe the patient's clinical data and outcome and also video-based details of our surgical method in removing the deeply embedded stinger. We will discuss the efficacy of our approach by comparing the features of our case with those of cases previously reported in the literature. Additionally, we suggest a general guideline for the management of corneal bee stings.

\section{Case report}

A 40-year-old Persian male was admitted to our emergency department 20 hours after his right eye was stung by a bee while driving. On arrival, the patient was complaining of severe pain, blurry vision, and tearing, which he had experienced soon after the injury. The right upper and lower eyelids were swollen, and significant conjunctival injection was present. His best-corrected visual acuity was 160/200 in the affected eye. Slit-lamp biomicroscopy revealed a bee stinger embedded into the cornea between an 8 o'clock and a 9 o'clock position. A $5 \times 5 \mathrm{~mm}^{2}$ corneal epithelial defect was notable at the site of the sting (Figure 1A-C). The intraocular pressure and depth of the anterior chamber were within normal limits. No anterior chamber reaction was observed. The lens was clear and the iris was normal. Examination was unremarkable in the left eye.
External removal of the stinger was not possible because the tip of the stinger was not accessible (Figure 1C). The patient was initially treated with a topical antibiotic (ciprofloxacin eye drops every 3 hours), a topical corticosteroid (fluorometholone eye drops every 8 hours), and a topical cycloplegic (tropicamide eye drops every 6 hours). Over the ensuing 2 days, corneal stromal infiltration and edema developed around the stinger site (Figure 1D-F), and visual acuity decreased to 100/200. Thereafter, surgical removal of the stinger was suggested (Figure 2). Under generalized anesthesia, an incision was made perpendicular to the corneal surface over the site of the sting. The (Video 1A)stinger was removed using a needle, (Video $1 \mathrm{~B}$ ) and smears of corneal scrapings were obtained for staining and culture. The corneal wound was sutured using a single 10-0 nylon suture (Video 1C). Finally, a subconjunctival injection of cefazolin $(50 \mathrm{mg} / \mathrm{mL})$ was given (Video 1D).

Postoperatively, the patient received topical ceftazidime and vancomycin eye drops. The tested culture was reported to be negative for bacteria and fungi. Subsequently, topical ciprofloxacin and tapering doses of betamethasone were replaced. This was maintained for the next 20 days. Of note, the suture was removed 14 days after surgery (Figure $1 \mathrm{G}$ and $\mathrm{H}$ ). Within approximately 30 days of follow-up, the corneal infiltration
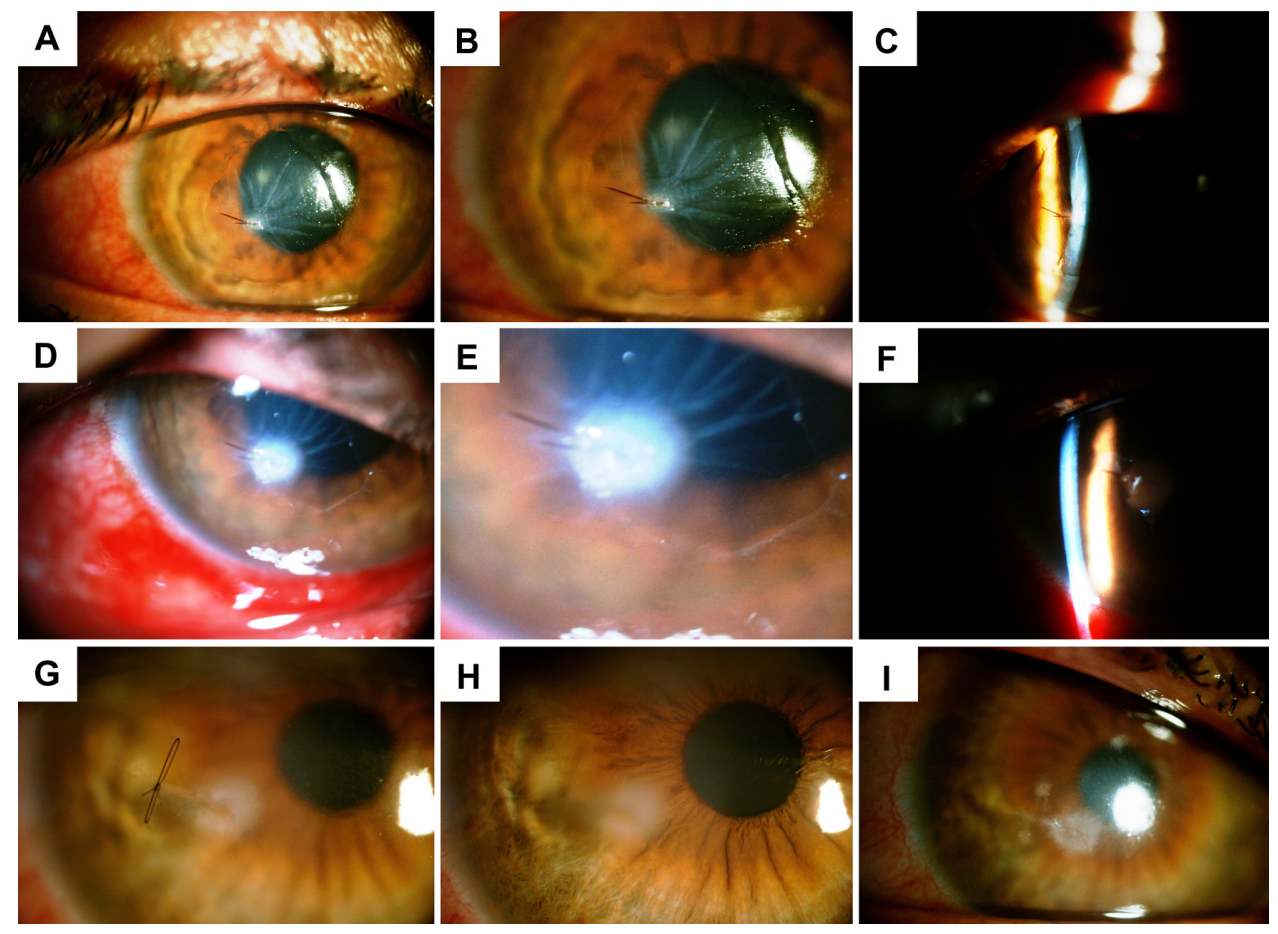

Figure I Photographs of the stung cornea in diffuse/slit illumination during approximately I month of follow-up. (A-C) Twenty hours after the injury: the deeply embedded stinger and the corneal epithelial defect are apparent around the site of injury. (D-F) Day 2 of follow-up (ie, the operation date). Note the corneal infiltration and edema, which led us to remove the stinger surgically. ( $\mathbf{G}$ and $\mathbf{H}$ ) Fourteen days after the operation: $(\mathbf{G})$ before suture removal; $(\mathbf{H})$ after suture removal. The infiltration is markedly reduced and the corneal epithelial defect is relatively healed. (I) One month after the operation: slight subepithelial scarring remains around the area of the stinger. 


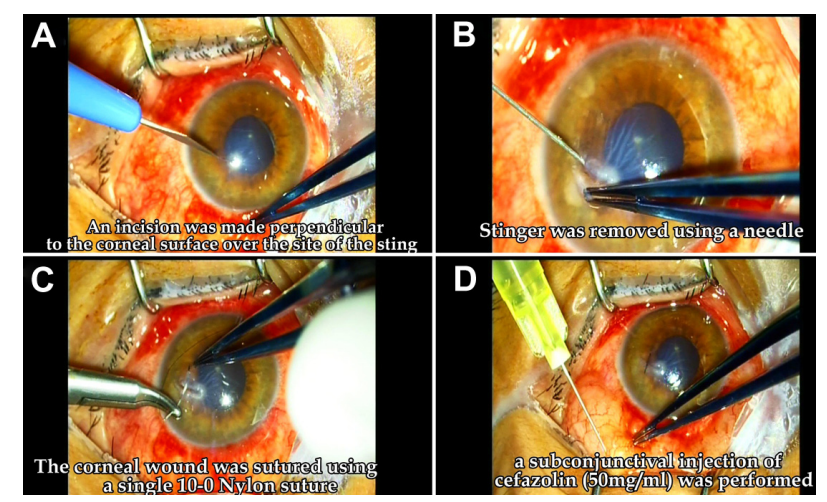

Figure 2 A demonstration of our surgical procedure (A-D: four steps) in removing the deeply embedded stinger on the second day of follow-up.

decreased gradually, and visual acuity improved to 180/200. However, slight subepithelial scarring remained around the area of the sting (Figure 1I).

\section{Discussion}

Here in this study we have described a patient with a deeply embedded bee stinger in the cornea. Our initial management included treatment with conventional drugs for eye injuries. However, complications (ie, stromal infiltration and edema) over the ensuing few days led us to choose a surgical approach to remove the stinger. In the literature, studies are controversial in respect of management strategies, depending on the severity of ocular complications and the status of the stinger within the eye. Several studies recommend initial therapy with topical antibiotics and corticosteroids for the prevention of secondary infection and suppression of venom-induced inflammation. ${ }^{4,5,10}$ Analgetics and cycloplegics were also reported to be beneficial in some instances. ${ }^{11}$ In a number of studies, the stinger was removed manually from the cornea in the first step by extracting its visible end from the cornea. ${ }^{2,11}$ However, external removal of the stinger can be difficult and is sometimes associated with the risk of leaving broken fragments within the cornea., ${ }^{4}$

Surgical removal of the retained bee stinger is still a matter of debate in the literature. ${ }^{4}$ Some authors suggest that surgical removal is not required in cases where the retained stinger is not associated with adverse ophthalmic complications. ${ }^{3,710}$ They postulate that once the venom from the bee stinger is neutralized, the stinger itself becomes completely inert and can remain within the cornea without causing further adverse reactions. In such studies, some cases were reported that the stinger remained within the cornea for many years asymptomatically. Gilboa et $\mathrm{al}^{7}$ addressed two patients with corneal bee sting in whom the stingers remained protruding into the anterior chamber for 21 years and 28 years with no ocular manifestations. Additionally, Arcieri et $\mathrm{al}^{3}$ described a 12-year-old boy with a retained corneal stinger protruding into the anterior chamber. He was treated with topical antibiotic and corticosteroids for a period of 1 month, which resulted in marked resolution of the symptoms. The authors indicated that the retained stinger had not caused any ocular complication and was completely reabsorbed within 2 years of follow-up. Another similar treatment was used by Chuah et $\mathrm{a}^{10}$ in a case with a retained stinger fragment in the cornea with no adverse results.

Aside from the aforementioned cases with conservative approaches, there are some instances in the literature in which retention of the stinger resulted in a severe corneal inflammation. Jain et al ${ }^{12}$ reported the case of a boy with a retained bee stinger that resulted in a state of kerato-uveitis. The stinger was removed surgically and symptoms improved. Moreover, Caça et $\mathrm{al}^{8}$ and Yildirim et al, ${ }^{9}$ in their studies, concisely addressed their surgical experience in removing deeply embedded stingers in patients who were complicated with corneal infiltration. Overall, it is generally accepted that immediate removal of the stinger is required in patients who are complicated with corneal infiltration and edema. ${ }^{12}$

In sum, leaving the stinger in place at initial stages depends on (1) severity of corneal reactions in the first hours (inflammation, infiltration, edema) and consequent visual outcome, (2) the distance of the stinger from the visual axis and consequent visual disturbances, (3) the ability of the physician to closely monitor over the ensuing weeks, and (4) most importantly, the depth of the protrusion and its external accessibility., ${ }^{7,10,12}$ Based on these factors, removal of the stinger at the slit lamp or in the operating room may be indicated and play a pivotal role in the management of corneal bee stings. In other words, stingers in the superficial cornea can be readily accessed and removed at the slit lamp. However, deeply embedded stingers, especially those extending to the anterior chamber, need surgery. Herein, the methodology and the experience of the surgeon can determine the final outcome.

In our case, the stinger was embedded into the cornea and could not be extracted externally. The patient was initially started with conventional treatment, including topical antibiotics, corticosteroids, and cycloplegics. Notwithstanding, after 2 days of initial therapy, corneal infiltration and edema appeared. Subsequently, surgical removal of the stinger was planned. Unfortunately, there are few recommendations on the precise procedure of surgical removal of the stinger in the literature. As can be seen from Video 1, we made an 
incision perpendicular to the corneal surface over the area of the sting and tried to extract the stinger using a needle. The stinger was removed completely, and the patient was given topical antibiotics and tapering doses of corticosteroids postoperatively. Our approach resulted in the resolution of corneal infiltration and improvement of visual acuity to an almost normal status. The patient is still on follow up, and, to date, no report of adverse outcome exists.

The majority of ophthalmic complications following corneal bee sting are suggested to be caused as a result of toxic or immunologic reactions to chemical mediators of the injected venom. ${ }^{2}$ However, in rare conditions, corneal bee sting is associated with infectious complications and bacterial culture growth. ${ }^{11}$ In our case, the culture result was negative, and the observed ocular complications might be caused by an immunologic reaction to the venom toxic materials.

In conclusion, we suggest a two-stage management approach for cases with corneal stingers. For the first stage, if (1) the stinger is readily accessible or (2) primary dramatic reactions (eg, infiltration), especially on the visual axis, exist, manual or surgical removal would be indicated. Otherwise, we recommend conventional treatments for eye injuries, including topical/systemic antibiotics, corticosteroids, and cycloplegics. Given this situation, patients should be closely monitored for the detection of any worsening. If the condition does not resolve or even deteriorates, for the second stage, surgical removal of the stinger under local or generalized anesthesia is indicated. However, the effectiveness of such an approach would be better clarified in more interventional studies.

\section{Acknowledgments}

Firstly, the authors are very grateful to Dr Alireza Peyman (Assistant Professor, IUMS) for his valuable comments and revisions. Secondly, we thank Mr Pouya Mir-Mohammmad-Sadeghi (student of medicine, IUMS) for his technical contribution, which was beyond any expressible value. Thirdly, we appreciate the efforts of Dr Ahmadreza Khalilian (resident of ophthalmology, IUMS) in monitoring the patient's status. Finally, we acknowledge Mr Khodadadi (camera man and picture editor of Feiz Hospital) and also, Mr Majid Fazeli (student of engineering, IUT). This study is dedicated to the memory of Dr Afsane Khandan (Iranian internist), who devoted her precious life to knowledge, health development, and medical research. This study was funded by "Sadra eye surgery centers" and also SHARNOS and Co. (Knowledge of Vision and Motion).

\section{Disclosure}

The authors have no proprietary interest in the materials presented herein.

\section{References}

1. Lin PH, Wang NK, Hwang YS, et al. Bee sting of the cornea and conjunctiva: management and outcomes. Cornea. 2011;30(4): 392-394.

2. Gürlü VP, Erda N. Corneal bee sting-induced endothelial changes. Cornea. 2006;25(8):981-983.

3. Arcieri ES, França ET, de Oliveria HB, et al. Ocular lesions arising after stings by hymenopteran insects. Cornea. 2002;21(3):328-330.

4. Teoh SC, Lee JJ, Fam HB. Corneal honeybee sting. Can J Ophthalmol. 2005;40(4):469-471.

5. Choi MY, Cho SH. Optic neuritis after bee sting. Korean J Ophthalmol. 2000;14(1):49-52.

6. Chinwattanakul S, Prabhasawat P, Kongsap P. Corneal injury by bee sting with retained stinger: a case report. J Med Assoc Thai. 2006;89(10): $1766-1769$.

7. Gilboa M, Gdal-On M, Zonis S. Bee and wasp stings of the eye. Retained intralenticular wasp sting: a case report. Br J Ophthalmol. 1977;61(10): 662-664.

8. Caça I, Ari S, Ulü K, Ayata A. Bee sting of the cornea: a case study and review of the literature. Ann Ophthalmol (Skokie). 2006;38(1):77-79.

9. Yildirim N, Erol N, Basmak H. Bee sting of the cornea: a case report. Cornea. 1998;17(3):333-334.

10. Chuah G, Law E, Chan WK, Ang CL. Case reports and mini review of bee stings of the cornea. Singapore Med J. 1996;37(4):389-391.

11. Smith DG, Roberge RJ. Corneal bee sting with retained stinger. J Emerg Med. 2001;20(2):125-128.

12. Jain V, Shome D, Natarajan S. Corneal bee sting misdiagnosed as viral keratitis. Cornea. 2007;26(10):1277-1278.
Clinical Ophthalmology

\section{Publish your work in this journal}

Clinical Ophthalmology is an international, peer-reviewed journal covering all subspecialties within ophthalmology. Key topics include: Optometry; Visual science; Pharmacology and drug therapy in eye diseases; Basic Sciences; Primary and Secondary eye care; Patient Safety and Quality of Care Improvements. This journal is indexed on Submit your manuscript here: http://www.dovepress.com/clinical-ophthalmology-journal

\section{Dovepress}

PubMed Central and CAS, and is the official journal of The Society of Clinical Ophthalmology (SCO). The manuscript management system is completely online and includes a very quick and fair peer-review system, which is all easy to use. Visit http://www.dovepress.com/ testimonials.php to read real quotes from published authors. 